\title{
Coexistence of localized and extended phases: Many-body localization in a harmonic trap
}

\author{
Titas Chanda $\odot,{ }^{1, *}$ Ruixiao Yao, ${ }^{2}$ and Jakub Zakrzewski $\oplus^{1,3, \dagger}$ \\ ${ }^{1}$ Institute of Theoretical Physics, Jagiellonian University in Kraków, Łojasiewicza 11, 30-348 Kraków, Poland \\ ${ }^{2}$ School of Physics, Peking University, Beijing 100871, China \\ ${ }^{3}$ Mark Kac Complex Systems Research Center, Jagiellonian University in Krakow, 30-348 Kraków, Poland
}

(Received 7 April 2020; revised 9 June 2020; accepted 28 July 2020; published 18 August 2020)

\begin{abstract}
We show that the presence of a harmonic trap may in itself lead to many-body localization for cold atoms confined in that trap in a quasi-one-dimensional geometry. Specifically, the coexistence of delocalized phase in the center of the trap with localized region closer to the edges is predicted with the borderline dependent on the curvature of the trap. The phenomenon, similar in its origin to Stark localization, should be directly observed with cold atomic species. We discuss both the spinless and the spinful fermions; for the latter we address Stark localization at the same time as it has not been analyzed up until now.
\end{abstract}

DOI: 10.1103/PhysRevResearch.2.032039

Introduction. For a long time, it has been believed that many-body systems tend to thermalize as expressed by eigenstate thermalization hypothesis [1,2]. The many-body localization (MBL) phenomenon (for reviews, see [3-6]) is a direct counterexample; for a sufficiently strong disorder, the system preserves the memory of its initial state. However, recent examples of many-body quantum so-called scar states [7,8], Hilbert-space fragmentation [9-12], and lack of thermalization in gauge theories [13-15] reveal strong nonergodic behaviors even in the absence of disorder. Another example considers Stark localization, where the presence of a static electric field resulting in a tilt in the many-body system may lead to localization $[16,17]$.

It seems that the more many-body physics is explored the less ergodic the many-body dynamics turns out to be. The present work provides another example of such a situation. We consider finite system sizes only. Such systems are directly amenable to experimental studies [18-27]. In this way we also stay away from a current vivid debate about the very existence of MBL in the thermodynamic limit [28-32]. We consider one-dimensional (1D) chains with chemical potentials quadratically dependent on position. Such a situation is quite common in quasi-1D situations realized in optical lattices $[33,34]$, where a tight confinement in directions perpendicular to a chosen one is due to illumination by strong laser beams with Gaussian transverse profiles. Those profiles may be well approximated as a harmonic trap along the considered direction [33]. Thus we shall consider models with

\footnotetext{
*titas.chanda@uj.edu.pl

†jakub.zakrzewski@uj.edu.pl

Published by the American Physical Society under the terms of the Creative Commons Attribution 4.0 International license. Further distribution of this work must maintain attribution to the author(s) and the published article's title, journal citation, and DOI.
}

Hamiltonians being

$$
H=H_{0}+H_{\text {trap }}=H_{0}+\frac{A}{2} \sum_{l=-L / 2}^{L / 2} l^{2},
$$

where $A$ is the curvature of the harmonic trap and $l$ is the site index (we assume unit spacing between sites of the chain). $H_{0}$ is the model Hamiltonian considered, which may represent the Heisenberg chain (equivalent to interacting spinless fermions), bosons represented by the Bose-Hubbard model, or spinful fermions with the Hubbard Hamiltonian. In contrast to the study of [17], where small quadratic potential has been considered on top of the dominant uniform linear potential, we consider the effect of a harmonic trap alone, which as we shall show acts differently in different parts of the system. Let us mention that such a model, for sufficiently big curvatures, may lead to a local quadruple conservation [9]; thus it belongs to a class of fracton systems where a generically slow subdiffusive approach to thermalization is expected [11,12]. For completeness, we mention that very slow dynamics was predicted also for harmonic trap quenches for the noninteracting case [35]. The results presented here are limited to moderate timescales where we observe no traces of the very slow thermalizing dynamics expected in the harmonic trap [11].

Heisenberg spins or spinless fermions. As the simplest possible model, first we consider the Heisenberg chain, where $H_{0}$ becomes

$$
H_{0}=J \sum_{l=-L / 2}^{L / 2-1} \vec{S}_{l} \cdot \vec{S}_{l+1}+\sum_{l=-L / 2}^{L / 2} h_{l} S_{l}^{z},
$$

with $\vec{S}_{l}$ 's being spin- $1 / 2$ operators and $h_{l}$ is a diagonal disorder (a magnetic field along the $z$ axis) drawn from random uniform distribution in the $[-W, W]$ interval. We set $J=1$ to be the unit of energy. The harmonic trapping potential in this case is given by $H_{\text {trap }}=\frac{A}{2} \sum_{l=-L / 2}^{L / 2} l^{2} S_{l}^{z}$. The Hamiltonian (2) is a paradigmatic model for MBL studies [36,37]; it maps to an interacting chain of spinless fermions via Jordan-Wigner 

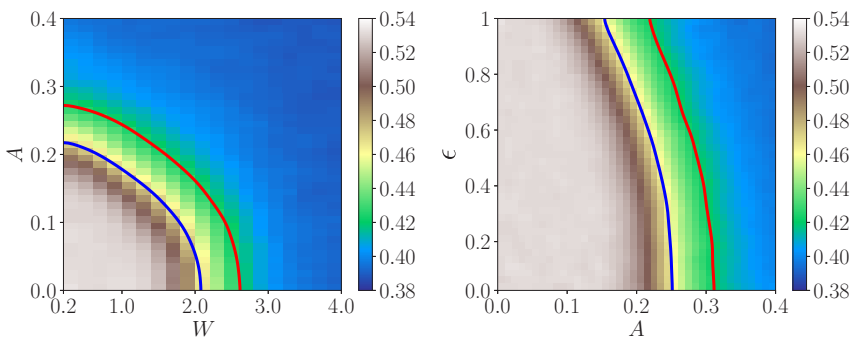

FIG. 1. Mean gap ratio $\bar{r}$ for the Heisenberg chain of $L=16$ sites. Left: $\bar{r}$ as a function of the disorder amplitude and curvature $A$. In the absence of external curvature $A=0$ the crossover to localized regime occurs for $W \approx 2.3$ for such a small system. Above $A=0.3$, the system seems, on the basis of mean gap ratio value, localized for arbitrary $W$. Right: $\bar{r}$ as a function of scaled energy $\epsilon$ and curvature $A$ at disorder strength $W=0.5$. The rescaled energy is defined as $\epsilon=\left(E-E_{\min }\right) /\left(E_{\max }-E_{\min }\right)$, where $E_{\min }\left(E_{\max }\right)$ is the energy of the ground (highest excited) state and $E$ denotes the energy of the corresponding eigenstate. The blue and red lines are contours for $\bar{r} \approx 0.46$ and 0.42 , respectively, and serve as a guide to the eyes for distinguishing apparently localized and ergodic phases.

transformation. A typical random matrix theory (RMT) based measure is the gap ratio defined as a minimum of the ratio of consecutive level spacings, $r_{n}=\min \left\{\frac{s_{n+1}}{s_{n}}, \frac{s_{n}}{s_{n+1}}\right\}$ with $s_{n}=$ $E_{n+1}-E_{n}$ and $E_{n}$ being the energy eigenvalue. The mean gap ratio is $\bar{r} \approx 0.53$ for a delocalized system, well described by Gaussian orthogonal ensemble (GOE), while $\bar{r} \approx 0.38$ for Poisson spectra characteristic for integrable, localized cases [38]. We find that for a sufficiently large curvature $A$ the mean gap ratio takes the latter value regardless of the disorder amplitude (see Fig. 1 for $L=16$ ). The figure resembles that observed for Stark localization [16].

To get insights into the physics observed, let us consider the time dynamics. We prepare the chain in the separable state with every second spin being up and down respectively as $|\uparrow, \downarrow, \uparrow, \ldots, \downarrow\rangle$ and observe whether this spin-wave arrangement is preserved in time evolution. For small disorder and small $A$ the system thermalizes (upper row in Fig. 2). For larger $A$ a different picture emerges; while at the center of the chain delocalization still occurs, at a sufficient distance from it we observe preservation of the initial spin texture, i.e., localization.

One can easily, a posteriori explain this phenomenon. For a given distance $l_{0}$ from the center the local static field can be expressed as $F=\frac{\partial}{\partial l_{0}}\left(\frac{A}{2} l_{0}^{2}\right)=l_{0} A$. If this local field exceeds the border of Stark localization [16,17], the part of the system localizes, while the region close to the center remains extended. Therefore, unlike the usual Stark localization, one can always find localized regions for any finite values of $A$ for large enough systems under harmonic trapping potential. The dashed lines in Fig. 2 give the Stark localization border, as predicted in [16] to be $F \approx 2$, which nicely fits numerical data.

While Fig. 2 clearly shows the coexistence of localized (close to edges) and delocalized (in the center of the trap) regions, this finding seems to be in contradiction with the mean gap ratio data of Fig. 1, which indicates that $\bar{r}$ takes the value close to Poissonian-like for $A=0.4$. Such a value may
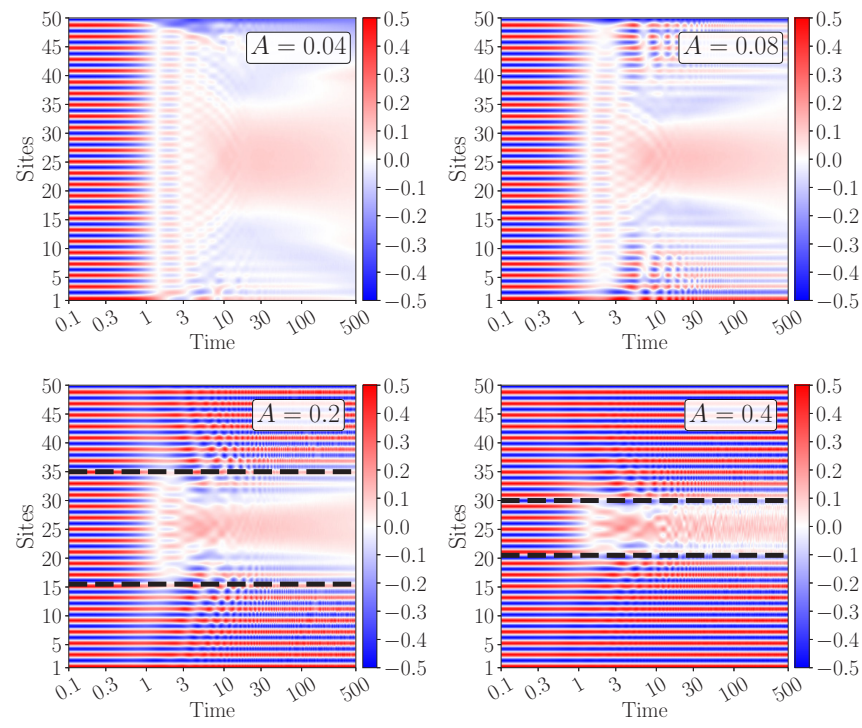

FIG. 2. Site-observed spin dynamics, as measured by the local expectation values $\left\langle S_{l}^{z}\right\rangle$ for the $L=50$ Heisenberg chain with no disorder. For small $A$ the system "thermalizes" and initial spin-wave configuration is destroyed by interactions. For larger curvature, one clearly observes the coexistence of delocalized (in the center) and localized regions (at the edges). The black dashed lines give the border of localization as given by Stark localization prediction with $F_{c} \approx 2[16]$.

correspond to a fully localized case, but also to a superposition of independent spectra. Therefore, a logical consequence is that the eigenstates are either localized close to the edges or extended over the central region, such that the mean gap ratio value comes as a result of the superposition of three independent spectra, only two of them being localized.

The simulations of time evolution are performed using a time-dependent variational principle (TDVP) algorithm using MPS ansatz [39-42]. More specifically, we use a hybrid variation of the TDVP scheme mentioned in [14,42-44], where we first use a two-site version of TDVP to dynamically grow the bond dimension up to a prescribed value, say $\chi_{\max }$. When the bond dimension in the MPS is saturated to $\chi_{\max }$, we shift to the one-site version to avoid any errors due to truncation in singular values that appears in the two-site version $[42,43]$. The final results are produced with $\chi_{\max }=512$, so that the maximum allowed value of the entanglement entropy at any given bond in the bulk of the system is $\ln \chi_{\max }=\ln (512)$.

Instead of spin profiles, one may look at the entanglement entropy growth in time (see Fig. 3). We see that entropy grows rapidly in the central region, while remaining low in the localized parts. In the delocalized center the entropy grows fast, and therefore, the simulations in this region may not be accurate. However, as we move towards the boundaries of the system, the results with $\chi_{\max }=512$ become "exact," even within the delocalized region. We confirm this by performing the same simulations with $\chi_{\max }=384$. The bottom row of Fig. 3 shows such a comparison of results with $\chi_{\max }=512$ and 384 for the $L=50$ Heisenberg chain with $A=0.2$ and 0.4 . Here, we only compare bonds that are in the delocalized part, as we always get converged results in the localized 

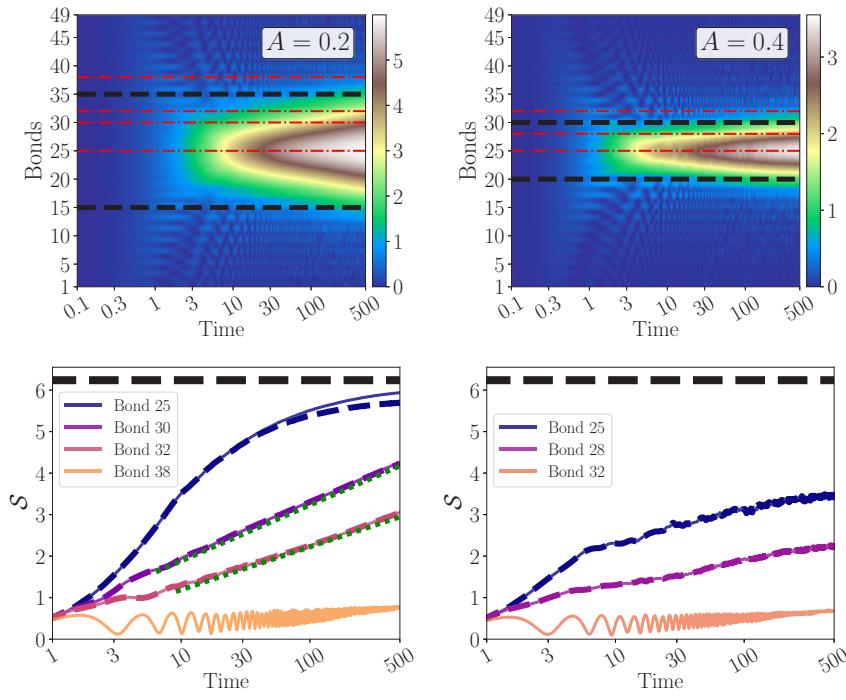

FIG. 3. Time dynamics of the entanglement entropy for $A=0.2$ (left column) and $A=0.4$ (right column) for the Heisenberg chain with the initial spin-wave state and without any disorder. Top row: Spatial profiles of time-evolved entropy measured across every bond. The entanglement grows rapidly in the central region remaining relatively low at the edges behind borders (depicted by black dashed lines) given by the Stark localization criterion. Bottom row: The growth of entanglement entropy with time measured across selected bonds (marked by red dash-dotted lines in the top row). Solid lines show the results for MPS bond dimension $\chi_{\max }=512$, while thicker dashed lines are for $\chi_{\max }=384$. The black dashed lines show the maximum allowed value $\ln 512$ of entanglement entropy by the MPS ansatz with $\chi_{\max }=512$ and the green dotted lines in the bottom left figure are straight lines in the logarithmic scale and are there as a guide to the eyes.

regions even for $\chi_{\max }=384$. The comparison of TDVP data with numerically exact results obtained using Chebyshev expansion of the time evolution operator [45-47] for $L=20$ is presented in [48].

Quite surprisingly, the entanglement entropy can also show logarithmic growth in time, even in the delocalized regions when the effect of trapping potential becomes strong. For example, in the case of $A=0.2$, entropy in the central bond grows rapidly in time and approaches the maximum allowed value by the MPS ansatz $[\ln (512)$ in this case]. On the other hand, the entropy in the bonds 30 and 32 shows a logarithmic growth, despite being on the delocalized side of the system. The entanglement growth in the case of $A=0.4$ is greatly modified by the harmonic trap even in the central bond. This is indeed very unusual dynamics where delocalized parts behave as systems showing MBL in terms of entropy growth. The plausible explanation of this behavior comes from the fact that entanglement entropies between nearby sites cannot differ much due to the local Hilbert space dimension being equal to 2 for spins. Thus logarithmic slow growth in time in the localized region also affects sites that are in the neighborhood of the border between localized and delocalized sites.

One important point to mention is that to observe clear signatures of MBL for the pure linear lattice tilt, either a small disorder or a slight curvature has to be added to
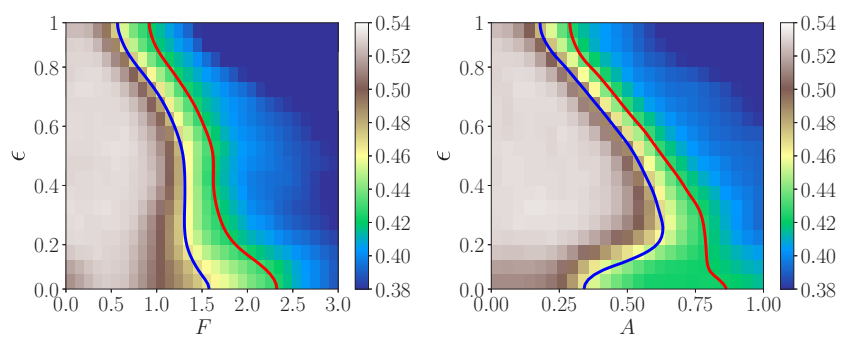

FIG. 4. Left: Gap ratio statistics for a static electric field problem for spinful fermions with interaction strength $U=1$ as a function of scaled energy $\epsilon$ and static field amplitude $F$ for disorder strength $W=0.5$. The plot corresponds to the six-fermion system in $L=$ 12 lattice sites (quarter filling) with the number of up and down fermions being equal. The transition to Poisson statistics is smooth and extended over a range of static field $F$ values. Stark localization is observed for sufficiently large $F$. Right: The gap ratio statistics for spinful fermions in the harmonic trap for disorder strength $W=0.5$. Other parameters are the same as in the left panel. The blue and red lines in both the figures are contours for $\bar{r} \approx 0.46$ and 0.42 , respectively, and serve as a guide to the eyes for distinguishing localized and ergodic phases.

the potential $[16,17]$ to avoid thermalization due to fracton dynamics [49]. For our harmonic potential the local field changes from site to site, which apparently suffices to avoid subdiffusive thermalization. Another interesting point is that the observed results do not warrant a finite-size scaling, as for a fixed value of $A$ the central region remains almost invariant and simply additional localized sites are added in the edges with increasing system size. For illustration, we put the results for $L=20$ in [48].

Spinful fermions: Stark localization. Since the interactions between spinless fermions are hard to realize experimentally in a standard cold atom in optical lattice setting, we consider the spinful case, represented by the Hubbard model, as in experiments [18,21]. The curvature-free part $H_{0}$ is

$$
H_{0}=-J \sum_{l, \sigma}\left(\hat{c}_{l \sigma}^{\dagger} \hat{c}_{l+1 \sigma}+\text { H.c. }\right)+U \sum_{l} \hat{n}_{l \uparrow} \hat{n}_{l \downarrow}+\sum_{l, \sigma} h_{l} \hat{n}_{l \sigma},
$$

with $l \in[-L / 2, L / 2]$. As before, we set $J=1$ to be the unit of energy and consider $U=1$ throughout this communication unless otherwise stated. Let us first consider the Stark localization problem under linear potential as it was only addressed for spinless fermions $[16,17]$ until now. Thus, we add to the Hamiltonian a tilt term $F \sum_{l} l\left(\hat{n}_{l \uparrow}+\hat{n}_{l \downarrow}\right)$ and analyze the gap ratio statistics. The corresponding $\bar{r}$ statistics is shown in Fig. 4(a) for the $L=12$ quarter-filled chain (i.e., $N_{\uparrow}=$ $N_{\downarrow}=L / 4$ ). To obtain this plot we break the SU(2) symmetry of the Hamiltonian by adding a local magnetic field to the Hamiltonian via the term $H_{\text {break }}=B\left(n_{L / 2 \uparrow}-n_{L / 2 \downarrow}\right)$ with $B=$ 0.5 following the prescription and discussion in [50]. We observe that, in comparison to spinless fermions [16], the crossover seems quite broad, possibly due to the small system size taken. To get a more precise critical value of $F$, we consider the time evolution of a staggered density-wave state $|\uparrow, 0, \downarrow, 0, \uparrow, 0, \downarrow, \ldots\rangle$ for larger system sizes and measure the density correlation $C(t)=D \sum_{l}\left[\bar{n}_{l}(t)-\rho\right]\left[\bar{n}_{l}(0)-\rho\right]$, where $\bar{n}_{l}(t)=\left\langle\hat{n}_{l \uparrow}+\hat{n}_{l \downarrow}\right\rangle, \rho$ is the average number of 

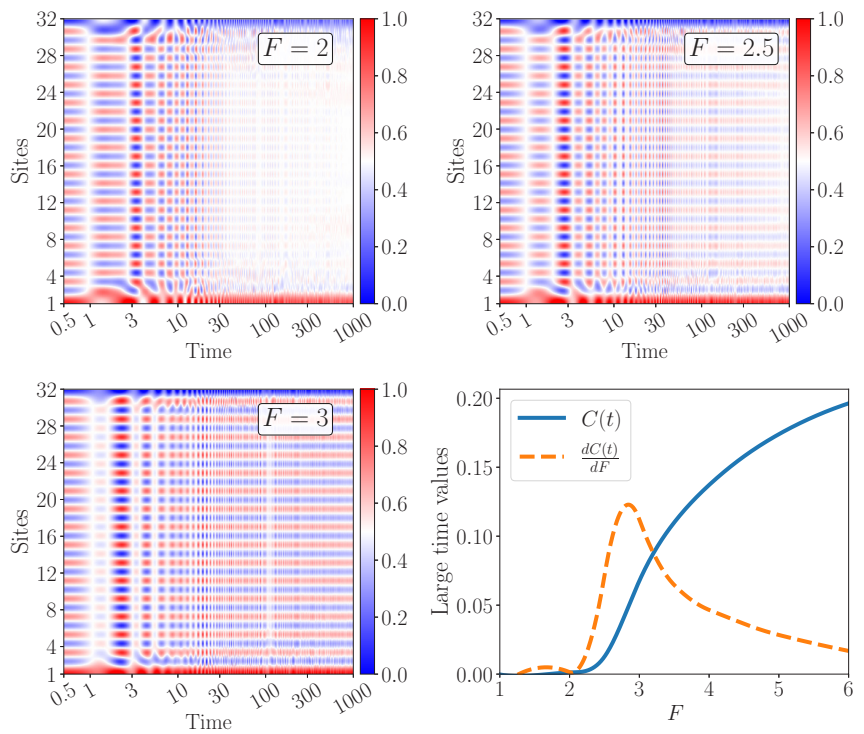

FIG. 5. Top row and bottom left panel: Time dynamics of spinful fermions for the initial staggered density-wave state without any disorder for three static field amplitudes, $F$, across the crossover. The localization is complete for $F=3$, while for intermediate field values a partial localization is observed with some fraction of particles accumulating near the bottom of the effective potential. Bottom right panel: Time-evolved density correlator $C(t)$ at large times as a function of $F$. We discard four sites from the boundaries to minimize their effects. We take the inflection point of the curve at $F_{c} \approx 2.8$ as a critical field amplitude. All data are for $L=32$ obtained using a TDVP algorithm.

particles per site, and the constant $D$ is chosen so that $C(0)=$ 1. The illustration of such time dynamics for the $L=32$ sites system is shown in Fig. 5. We observe that for the high field value, e.g., $F=3$, the localization is almost complete (bottom left panel). On the other hand, at lower fields, we observe a partial localization [as revealed also by standard local densities $\bar{n}_{l}(t)$; see top row of Fig. 5]. The bottom right panel of Fig. 5 shows large time values of the density correlator $C(t)$ and its derivative $\frac{d C(t)}{d t}$. We approximate critical $F_{c}$ from the inflection point of $\stackrel{d t}{C}(t)$, as obtained from the maximal value of its derivative, to be $F_{c} \approx 2.8$ for the disorderless scenario.

Spinful fermions: Localization under harmonic trap. Having established the estimate for the critical field amplitude corresponding to the crossover to localized phase, we may turn again to the harmonic confinement case. Thus we again consider (1) now for spinful fermions (3), with $H_{\text {trap }}=$ $\frac{A}{2} \sum_{l=-L / 2}^{L / 2} l^{2}\left(\hat{n}_{l \uparrow}+\hat{n}_{l \downarrow}\right)$. The right panel of Fig. 4 shows level spacing statistics for the quarter-filled $L=12$ chain in such case and Fig. 6 depicts the time dynamics of the density profile for different curvatures of the harmonic potential with the staggered density-wave state being the initial one. We observe that, as in the spinless fermions case, while in the center of the trap apparent fast "thermalization" occurs, closer to the edges the effective electric field coming from the curvature of the trap leads to localization. The dashed lines give the estimate of the threshold assuming the $F=l_{0} A$ condition with $F_{c}=2.8$ from the previous analysis.
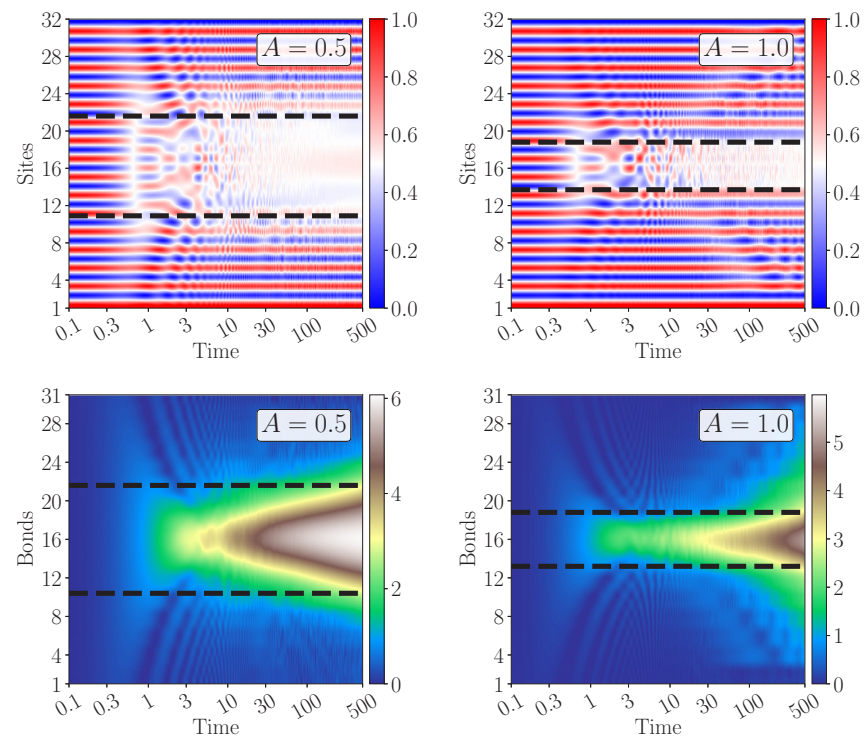

FIG. 6. Top row: Time-evolved density profile of spinful fermions for the initial staggered density-wave state under harmonic potential with no disorder. Bottom row: Time dynamics of entanglement entropy measured across every bond for the same systems as in the top row. All the figures are for the quarter-filled $L=32$ chain. Black dashed lines give the physical border of localization as predicted by the Stark localization with $F_{c} \approx 2.8$.

We may also analyze the time-evolved entanglement entropy in different regions. While in the center of the trap the entropy grows linearly with time and soon saturates due to insufficient bond dimension of the MPS ansatz rendering the results in this region not accurate, the entropy beyond the localization boundary given by $l_{0}>F / A$ (and symmetrically for negative $l_{0}$ ) seems to grow logarithmically providing further evidence for many-body localization in the outer regions.

The corresponding spin dynamics is also interesting. As in the standard MBL case, we observe a subdiffusive decay of initial spin configuration for the initial staggered density-wave state, characteristic of the remaining SU(2) symmetry of the problem [51-53]. Visualization of this effect can be seen from the profile of spin degrees of freedom in Fig. 7, where a slow spreading of the delocalized region in the spin sector at later times can be observed.
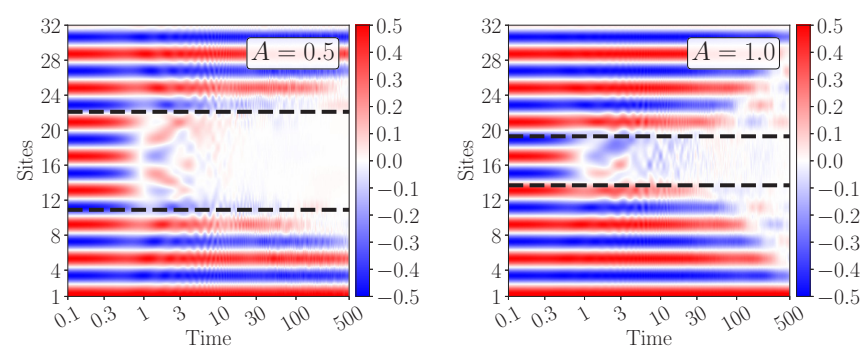

FIG. 7. Time-evolved profile of local spin operator $S_{l}^{z}=\left(\hat{n}_{l \uparrow}-\right.$ $\left.\hat{n}_{l \downarrow}\right) / 2$ of spinful fermions for the initial staggered density-wave state under harmonic potential with no disorder. Other specifications are the same as in Fig. 6. 
Conclusions. We have shown that many-body localization behavior can be observed in the presence of the harmonic trap and in the absence of the disorder. The effect is due to a local static field that induces, for sufficient curvatures, Stark localization as recently shown for spinless fermions $[16,17,49]$ and announced in the spinful case [54]. Since the effect has a lower bound on the curvature, the central region of the trap remains delocalized. Thus a harmonic trap makes a possible realization of a very interesting situation-coexistence of delocalized and MBL phases in a single system. Let us stress that on the experimentally relevant timescales considered by us, we do not observe any traces of the slow subdiffusive thermalization predicted due to fracton hydrodynamics [11]. Finally, let us also mention that the harmonic trap may play some role in the experiments on MBL performed in optical lattices (e.g., [18,21]) as the residual trap due to Gaussian beam profiles is most probably present there. While in these experiments disorder-induced effects play a dominant role, the residual harmoniclike trap may affect the details of the time dynamics for large systems. This aspect is the subject of a current research. Finally, we note that the effect is not limited to harmonic trap but can be generalized to arbitrary potentials with nonvanishing first-order derivatives.

Acknowledgments. The numerical computations have been possible thanks to High-Performance Computing Platform of Peking University as well as PL-Grid Infrastructure. The TDVP simulations have been performed using the ITensor library [55]. This research has been supported by National Science Centre (Poland) under projects 2017/25/Z/ST2/ 03029 (T.C.) and 2016/21/B/ST2/01086 (J.Z.)
[1] J. M. Deutsch, Phys. Rev. A 43, 2046 (1991).

[2] M. Srednicki, Phys. Rev. E 50, 888 (1994).

[3] D. A. Huse, R. Nandkishore, and V. Oganesyan, Phys. Rev. B 90, 174202 (2014).

[4] R. Nandkishore and D. A. Huse, Annu. Rev. Condens. Matter Phys. 6, 15 (2015).

[5] F. Alet and N. Laflorencie, C. R. Phys. 19, 498 (2018).

[6] D. A. Abanin, E. Altman, I. Bloch, and M. Serbyn, Rev. Mod. Phys. 91, 021001 (2019).

[7] C. J. Turner, A. A. Michailidis, D. A. Abanin, M. Serbyn, and Z. Papić, Nat. Phys. 14, 745 (2018).

[8] A. J. A. James, R. M. Konik, and N. J. Robinson, Phys. Rev. Lett. 122, 130603 (2019).

[9] V. Khemani, M. Hermele, and R. Nandkishore, Phys. Rev. B 101, 174204 (2020).

[10] P. Sala, T. Rakovszky, R. Verresen, M. Knap, and F. Pollmann, Phys. Rev. X 10, 011047 (2020).

[11] A. Gromov, A. Lucas, and R. M. Nandkishore, Phys. Rev. Research 2, 033124 (2020).

[12] J. Feldmeier, P. Sala, G. de Tomasi, F. Pollmann, and M. Knap, arXiv:2004.00635.

[13] M. Brenes, M. Dalmonte, M. Heyl, and A. Scardicchio, Phys. Rev. Lett. 120, 030601 (2018).

[14] T. Chanda, J. Zakrzewski, M. Lewenstein, and L. Tagliacozzo, Phys. Rev. Lett. 124, 180602 (2020).

[15] G. Magnifico, M. Dalmonte, P. Facchi, S. Pascazio, F. V. Pepe, and E. Ercolessi, Quantum 4, 281 (2020).

[16] E. van Nieuwenburg, Y. Baum, and G. Refael, Proc. Natl. Acad. Sci. USA 116, 9269 (2019).

[17] M. Schulz, C. A. Hooley, R. Moessner, and F. Pollmann, Phys. Rev. Lett. 122, 040606 (2019).

[18] M. Schreiber, S. S. Hodgman, P. Bordia, H. P. Lüschen, M. H. Fischer, R. Vosk, E. Altman, U. Schneider, and I. Bloch, Science 349, 842 (2015).

[19] J. Smith, A. Lee, P. Richerme, B. Neyenhuis, P. W. Hess, P. Hauke, M. Heyl, D. A. Huse, and C. Monroe, Nat. Phys. 12, 907 (2016).

[20] P. Roushan et al., Science 358, 1175 (2017).

[21] H. P. Lüschen, P. Bordia, S. Scherg, F. Alet, E. Altman, U. Schneider, and I. Bloch, Phys. Rev. Lett. 119, 260401 (2017).

[22] D. M. Silevitch, C. Tang, G. Aeppli, and T. F. Rosenbaum, Nat. Commun. 10, 4001 (2019).
[23] K. X. Wei, C. Ramanathan, and P. Cappellaro, Phys. Rev. Lett. 120, 070501 (2018).

[24] K. Xu, J.-J. Chen, Y. Zeng, Y.-R. Zhang, C. Song, W. Liu, Q. Guo, P. Zhang, D. Xu, H. Deng, K. Huang, H. Wang, X. Zhu, D. Zheng, and H. Fan, Phys. Rev. Lett. 120, 050507 (2018).

[25] Q. Guo, C. Cheng, Z.-H. Sun, Z. Song, H. Li, Z. Wang, W. Ren, H. Dong, D. Zheng, Y.-R. Zhang, R. Mondaini, H. Fan, and H. Wang, arXiv:1912.02818.

[26] A. Lukin, M. Rispoli, R. Schittko, M. E. Tai, A. M. Kaufman, S. Choi, V. Khemani, J. Léonard, and M. Greiner, Science 364 256 (2019).

[27] M. Rispoli, A. Lukin, R. Schittko, S. Kim, M. E. Tai, J. Léonard, and M. Greiner, Nature (London) 573, 385 (2019).

[28] J. Šuntajs, J. Bonča, T. Prosen, and L. Vidmar, arXiv: 1905.06345.

[29] D. A. Abanin, J. H. Bardarson, G. D. Tomasi, S Gopalakrishnan, V. Khemani, S. A. Parameswaran, F. Pollmann, A. C. Potter, M. Serbyn, and R. Vasseur, arXiv:1911.04501.

[30] P. Sierant, D. Delande, and J. Zakrzewski, Phys. Rev. Lett. 124, 186601 (2020).

[31] R. K. Panda, A. Scardicchio, M. Schulz, S. R. Taylor, and M. Žnidarič, Europhys. Lett. 128, 67003 (2020).

[32] P. Sierant, M. Lewenstein, and J. Zakrzewski, arXiv:2005.09534.

[33] L. Fallani, J. E. Lye, V. Guarrera, C. Fort, and M. Inguscio, Phys. Rev. Lett. 98, 130404 (2007).

[34] J. Zakrzewski and D. Delande, Phys. Rev. A 80, 013602 (2009).

[35] M. Schulz, C. A. Hooley, and R. Moessner, Phys. Rev. A 94, 063643 (2016).

[36] D. J. Luitz, N. Laflorencie, and F. Alet, Phys. Rev. B 91, 081103(R) (2015)

[37] P. Sierant and J. Zakrzewski, Phys. Rev. B 99, 104205 (2019).

[38] V. Oganesyan and D. A. Huse, Phys. Rev. B 75, 155111 (2007).

[39] J. Haegeman, J. I. Cirac, T. J. Osborne, I. Pižorn, H. Verschelde, and F. Verstraete, Phys. Rev. Lett. 107, 070601 (2011).

[40] T. Koffel, M. Lewenstein, and L. Tagliacozzo, Phys. Rev. Lett. 109, 267203 (2012).

[41] J. Haegeman, C. Lubich, I. Oseledets, B. Vandereycken, and F. Verstraete, Phys. Rev. B 94, 165116 (2016).

[42] S. Paeckel, T. Köhler, A. Swoboda, S. R. Manmana, U. Schollwöck, and C. Hubig, Ann. Phys. 411, 167998 (2019). 
[43] S. Goto and I. Danshita, Phys. Rev. B 99, 054307 (2019).

[44] T. Chanda, P. Sierant, and J. Zakrzewski, arXiv:2006.02860.

[45] H. Tal-Ezer and R. Kosloff, J. Chem. Phys. 81, 3967 (1984).

[46] C. Leforestier, R. Bisseling, C. Cerjan, M. Feit, R. Friesner, A. Guldberg, A. Hammerich, G. Jolicard, W. Karrlein, H.-D. Meyer, N. Lipkin, O. Roncero, and R. Kosloff, J. Comput. Phys. 94, 59 (1991).

[47] H. Fehske and R. Schneider, Computational Many-Particle Physics (Springer, Germany, 2008).

[48] See Supplemental Material at http://link.aps.org/supplemental/ 10.1103/PhysRevResearch.2.032039 for additional results.
[49] S. R. Taylor, M. Schulz, F. Pollmann, and R. Moessner, arXiv:1910.01154.

[50] R. Mondaini and M. Rigol, Phys. Rev. A 92, 041601(R) (2015).

[51] P. Prelovšek, O. S. Barišić, and M. Žnidarič, Phys. Rev. B 94, 241104(R) (2016).

[52] M. Środa, P. Prelovšek, and M. Mierzejewski, Phys. Rev. B 99, 121110(R) (2019).

[53] J. Zakrzewski and D. Delande, Phys. Rev. B 98, 014203 (2018).

[54] T. Kohlert, S. Scherg, B. H. Madhusudhana, I. Bloch, and M. Aidelsburger, Bull. Am. Phys. Soc. (2020), http://meetings.aps. org/Meeting/DAMOP20/Session/D09.9.

[55] https://itensor.org. 